\title{
Risk Factors for Cerebral Infarction After Moderate or Severe Traumatic Brain Injury
}

\author{
Yin-gang $\mathrm{Wu}^{1,2}$ \\ Yingjiu Chao' \\ Ge Gao' \\ Dejun Bao' \\ Yongfei Dong' \\ Xiangpin Wei ${ }^{1}$ \\ Chaoshi Niu ${ }^{1,2}$
}

'Department of Neurosurgery, The First Affiliated Hospital of USTC, Division of Life Sciences and Medicine, University of Science and Technology of China, Hefei, Anhui, 23000I, People's Republic of China; ${ }^{2}$ Brain Function and Brain Disease Anhui Provincial Key Laboratory, Hefei, Anhui, 23000I, People's Republic of China

Correspondence: Chaoshi Niu Department of Neurosurgery, The First Affiliated Hospital of USTC, Division of Life Sciences and Medicine, University of Science and Technology of China, Hefei, Anhui, 23000I, People's Republic of China Tel +8618963788788

Email niuchaoshi@।26.com
Purpose: Posttraumatic cerebral infarction (PTCI) is a common and relatively serious complication of traumatic brain injury (TBI) without a clear etiology. Evaluating risk factors in advance is particularly important to predict and avoid the occurrence of PTCI.

Patients and Methods: We retrospectively analyzed 297 patients with moderate to severe TBI admitted to the Department of Neurosurgery in our hospital from January 2019 to September 2020 and evaluated the effects of various factors such as age, sex, admission Glasgow Coma Scale (GCS), skull base fracture, subarachnoid hemorrhage (SAH), brain herniation, hypotensive shock, and decompressive craniectomy on the incidence of PTCI. We also performed a multivariate logistics regression analysis on the relevant factors identified and evaluated the diagnostic value of each risk factor in advance by receiver operating characteristic (ROC) analyses.

Results: Among the patients, 32 (10.77\%) suffered PTCI. The incidence rates of PTCI in those with GCS scores of 3-8 and 9-12 were $15.87 \%(30 / 189)$ and $1.85 \%(2 / 108)$, respectively, while the rates were $18.84 \%$ (13/69), $15.03 \%$ (29/193), $18.57 \%(13 / 70)$, and $20.59 \%$ $(14 / 68)$ in those with skull base fractures, traumatic SAH, brain herniation, and hypotensive shock, respectively, and $14.38 \%(23 / 160)$ in those who underwent decompressive craniectomy. These differences in PTCI incidence were statistically significant. However, the differences in PTCI incidence caused by patient age and sex were not statistically significant. Conclusion: Low GCS score, skull base fractures, traumatic SAH, brain herniation, hypotensive shock, and decompressive craniectomy are risk factors for the occurrence of PTCI, while age and sex are not significantly correlated with the occurrence of PTCI.

Keywords: moderate or severe traumatic brain injury, posttraumatic cerebral infarction, risk factor

\section{Introduction}

Posttraumatic cerebral infarction (PTCI) is a common secondary posttraumatic injury and often leads to poor prognosis, but the mechanism of its occurrence is not yet clear. Assessing risk factors in advance is very important to prevent the occurrence of PTCI. Previously, direct compression of the blood supply artery in the infarcted area was believed to be the main cause of PTCI. However, some patients with significant mass effects do not develop PTCI, while some patients with less significant mass effects develop PTCI unexpectedly, suggesting that some other factors may determine the occurrence of PTCI.

In this study, we retrospectively analyzed the risk factors of PTCI based on the data of 297 patients with moderate to severe TBI admitted to the Department of Neurosurgery, The First Affiliated Hospital of USTC (University of Science and Technology of China) from January 2019 to September 2020 to provide a reference for future clinical work. 


\section{Materials and Methods}

\section{Patients and Methods}

We retrospectively analyzed patients with moderate to severe TBI who were admitted to the Department of Neurosurgery in our hospital between January 2019 and September 2020. A total of 297 patients were identified in this study and the criteria involved were as followed (Figure 1).

\section{Inclusion Criteria}

(1) moderate to severe TBI patients with admission to the Department of Neurosurgery of our hospital from January 2019 to September 2020; (2) complete clinical data; and (3) complete follow-up data.

\section{Exclusion Criteria}

(1) a previous history of cerebral infarction and severe heart diseases; (2) poorly controlled hypertension, hyperlipidemia, or diabetes; (3) death within 3 days after admission; (4) a GCS score above 12 points; and (5) severe fractures and pulmonary contusions.

\section{General Information}

Among the patients, 190 were males and 107 females, with ages ranging from 11 months to 90 years (average 37.4 years). The admission Glasgow coma scale (GCS) scores were as follows: 3-8 points: 189 cases; and 9-12 points: 108 cases. The following complications were observed: skull base fracture: 69 cases; traumatic SAH: 193 cases; cerebral herniation: 70 cases; and hypotensive shock: 68 cases.

\section{Patient Treatment}

All patients were treated in accordance with the 2007 guidelines for the management of severe traumatic brain injury (TBI). ${ }^{1}$ A total of 160 patients underwent decompressive craniectomy, 2 of whom received a second decompressive craniectomy after secondary large-area traumatic cerebral infarction.

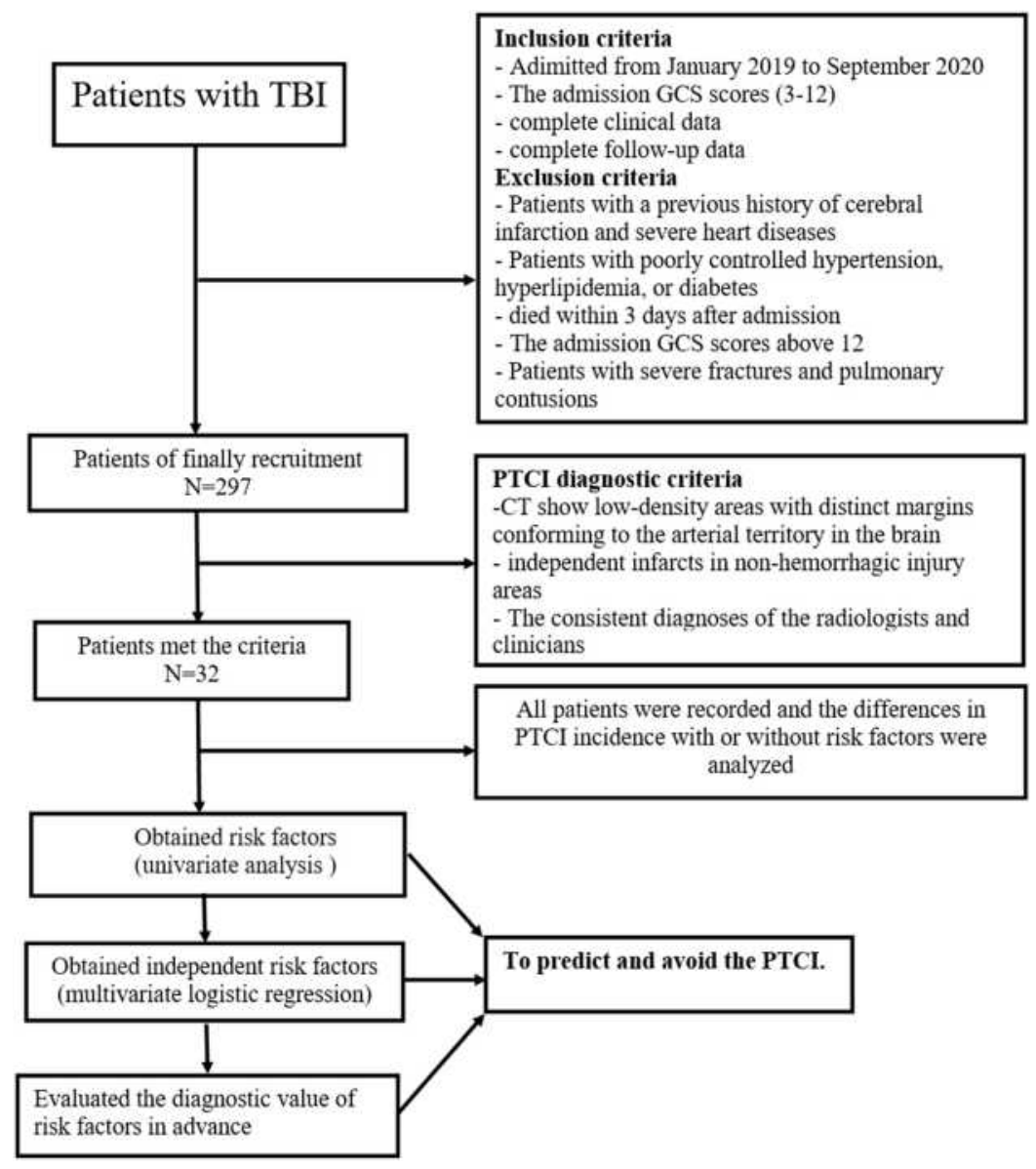

Figure I The experimental flow chart and criteria involved. 
PTCI Diagnostic Criteria: ${ }^{2}$ Multiple computed tomography $(\mathrm{CT})$ examinations after TBI showing low-density areas with distinct margins conforming to the arterial territory in the brain meeting the following conditions: independent infarcts in non-hemorrhagic injury areas; the consistent diagnoses of the radiologists and clinicians. While the focal cerebral or cortical encephalomalacia after craniectomy and obvious exogenous embolic factors, such as air embolism, lipid embolism, or cardiogenic embolism were excluded.

\section{Statistical Analysis}

The comparison of two groups was analyzed by $\chi^{2}$ test (or Fisher exact test) for univariate analysis for each variable. The logistic regression model was used for multivariate analysis to detect independent risk factors for the onset of PTCI. Variables with $\mathrm{P}$ value $<0.05$ determined by univariate analysis were included for multivariate analysis. In the final multivariate logistic regression model, variables with $\mathrm{P}$ values $<0.05$ were considered statistically significant. A receiver operating characteristic (ROC) curve was created from independent risk factors obtained from the multivariable logistic regression analysis. The area under the curve (AUC), 95\% confidence interval (CI) and cut-off value of the continuous variables were recorded. The ROC model was analyzed with MedCalc statistical software (version 19.0.4, Ostend, Belgium) and the statistical package Statistical Analysis System for Windows (version 20.0, SAS Institute, Cary, NC, USA) was used for analyses.

\section{Results}

\section{Patient Characteristics}

A total of $32(10.77 \%)$ patients with PTCI were included, 11 of whom (34.38\%) had PTCI within 1 week, $16(50 \%)$ within 8-14 days, 2 (6.25\%) within 14-21 days, 2 (6.25\%) within 21 days to 1 month, and $1(3.12 \%)$ within 1 to 3 months. Of the 32 patients with PTCI, 23 had 2 or more infarcts in the vascular blood supply area, and 9 had a single infarct in the vascular blood supply area; 21 patients had infarcts in the blood supply area of the posterior cerebral artery (PCA), 21 in the blood supply area of the middle cerebral artery (MCA),
7 in the blood supply area of the anterior cerebral artery (ACA), 6 in the basal ganglia area, and 6 in the posterior fossa. The prognoses at 6 months after injury were as follows: among the 297 patients, 194 had a good prognosis (Good recovery/moderate disability), and 103 had a poor prognosis (Severe disability/vegetative/death); among the 32 patients with PTCI, 12 a had good prognosis, and 20 had a poor prognosis (Table 1).

\section{Univariable Analysis}

The risk factors analysis for PTCI in 297 patients with TBI showed that the admission GCS, skull base fractures, traumatic SAH, brain herniation, hypotensive shock, and decompressive craniectomy were associated with PTCI in patients with moderate or severe TBI, whereas gender and age were not (Table 2).

\section{Multivariable Logistic Regression Analysis}

The multivariate analysis identified that low admission, skull base fractures, traumatic SAH, brain herniation, hypotensive shock, and decompressive craniectomy were risk factor for PTCI in patients with moderate or severe TBI. No association was observed between PTCI and gender or age (Table 3).

\section{ROC Curve Analysis}

The independent risk factors obtained from the multivariable logistic regression model were entered into the ROC model. Figure 2 presents the ROC curves of the six independent risk factors. Table 4 presents the characteristics in the ROC curves of the six independent risk factors.

\section{Discussion}

The prognosis of patients with head injuries is closely associated with secondary injury. It is also seriously affected by PTCI, one of the common and serious complications of TBI. Previously, Mirvis et $\mathrm{al}^{2}$ reported that the incidence of PTCI is $1.9 \%$. The development of medical imaging techniques such as $\mathrm{CT}$ and magnetic resonance imaging (MRI) has facilitated PTCI diagnosis and significantly increased the detection rate of PTCI. According to the latest report, ${ }^{3}$ the incidence of PTCI is $9.7 \%$. Our statistics indicated that the incidence is $10.77 \%$,

Table I The Prognosis of 297 Patients 6 Months After Injuried

\begin{tabular}{|l|c|c|}
\hline Outcome at 6 Months & With PTCI (32 Patients) & Without PTCI (265 Patients) \\
\hline Good recovery/moderate disability & $12(37.5 \%)$ & $182(68.68 \%)$ \\
Severe disability/vegetative/death & $20(62.5 \%)$ & $83(31.32 \%)$ \\
\hline
\end{tabular}

Note: All correlation comparisons showed a significance level of $P<0.05$. 
Table 2 The Risk Factors for PTCl in 297 Patients with Moderate or Severe TBI

\begin{tabular}{|c|c|c|c|}
\hline Factor & No. of Patients & PTCI & $P$ value \\
\hline Gender & & & 0.683 \\
\hline Male & 190 & $22(11.58 \%)$ & \\
\hline Female & 107 & $10(9.35 \%)$ & \\
\hline Age (years) & & & 0.702 \\
\hline$<20$ & 77 & 9 (11.68\%) & \\
\hline $20-40$ & 159 & $17(10.69 \%)$ & \\
\hline$>40$ & 61 & $6(9.84 \%)$ & \\
\hline Admission GCS & & & 0.000 \\
\hline $3-8$ & 189 & $30(15.87 \%)$ & \\
\hline $9-12$ & 108 & $2(1.85 \%)$ & \\
\hline Skull base fractures & & & 0.007 \\
\hline Yes & 69 & $13(18.84 \%)$ & \\
\hline No & 228 & $19(8.33 \%)$ & \\
\hline Traumatic SAH & & & 0.004 \\
\hline Yes & 193 & $29(15.03 \%)$ & \\
\hline No & 104 & $3(2.88 \%)$ & \\
\hline Brain herniation & & & 0.029 \\
\hline Yes & 70 & $13(18.57 \%)$ & \\
\hline No & 227 & $19(8.37 \%)$ & \\
\hline Hypotensive shock & & & 0.000 \\
\hline Yes & 68 & 14 (20.59\%) & \\
\hline No & 229 & $18(7.86 \%)$ & \\
\hline Decompressive craniectomy & & & 0.034 \\
\hline Yes & 160 & $23(14.38 \%)$ & \\
\hline No & 137 & 9 (6.57\%) & \\
\hline
\end{tabular}

Note: All correlation comparisons showed a significance level of $P<0.05$.

Table 3 The Outcome of the Multivariate Logistic Regression Analysis of the Association Between Risk Factors and PTCl

\begin{tabular}{|l|c|c|c|}
\hline Factors & OR value & $\mathbf{9 5 \%} \mathbf{~ C I}$ & P value \\
\hline Gender & $1.27 \mathrm{I}$ & $0.527-2.232$ & 0.458 \\
\hline Age(years) & 0.768 & $0.426-1.429$ & 0.432 \\
\hline Admission GCS & 10.324 & $2.763-17.773$ & $0.00 \mathrm{I}$ \\
\hline Skull base fractures & 2.553 & $1.723-4.846$ & $0.03 \mathrm{I}$ \\
\hline Traumatic SAH & 5.935 & $1.475-8.617$ & 0.027 \\
\hline Brain herniation & 2.497 & $\mathrm{I} .164-5.013$ & 0.036 \\
\hline Hypotensive shock & 3.034 & $1.40 \mathrm{I}-5.848$ & $0.00 \mathrm{I}$ \\
\hline $\begin{array}{l}\text { Decompressive } \\
\text { craniectomy }\end{array}$ & $2.38 \mathrm{I}$ & $\mathrm{I} .164-5.01 \mathrm{I}$ & $0.04 \mathrm{I}$ \\
\hline
\end{tabular}

Note: All correlation comparisons showed a significance level of $P<0.05$. Abbreviations: OR, odds ratio; $\mathrm{Cl}$, confidence interval. which is slightly higher than $9.7 \%$, likely due to our exclusion of patients with an admission GCS score greater than 12 and patients who died within 3 days after admission. Latronico et $\mathrm{al}^{3}$ reported that the mortality or disability rate of TBI patients with PTCI is $67.4 \%$, which is considerably higher than that of patients without PTCI (28.4\%). Our findings are essentially consistent with Latronico's results, that is, the 6-month mortality or disability rate of TBI patients with PTCI is $62.50 \%$, and that of TBI patients without PTCI is $31.32 \%(\mathrm{p}<0.01)$.

PTCI often occurred in the early stage of TBI (10 days on average), and 27 cases (84.38\%) occurred within 2 weeks. Therefore, CT is recommended within 2 weeks after injury such that PTCI can be detected and treated early. Our data showed that the incidence of PTCI in the blood supply area of the PCA ( 21 cases) or the MCA (21 cases) is significantly higher than that in other areas, followed by that of the ACA (7 cases), which is consistent with the rate previously 


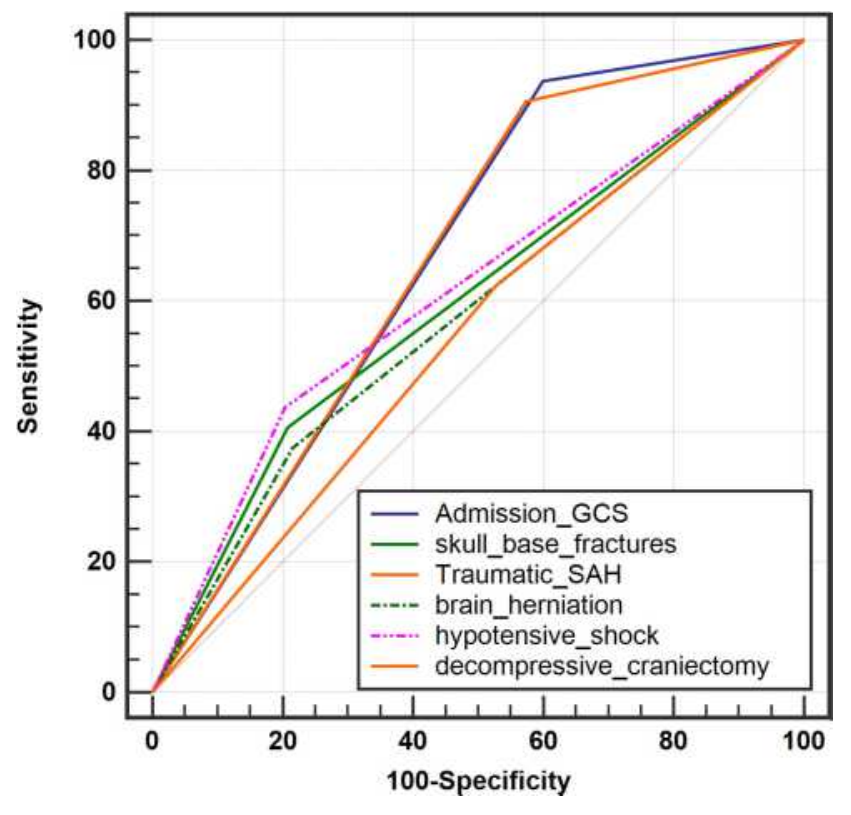

Figure 2 ROC model of the six independent risk factors obtained from the multivariate logistic regression analysis.

Abbreviations: GCS, Glasgow Coma Scale; SAH, subarachnoid hemorrhage.

reported by Liu et al, ${ }^{4}$ suggesting that PTCI likely occurs because these vessels are more likely to be compressed and thus deformed. The PCA is susceptible to be compressed by the temporal lobe sulcus, which leads to stenosis or even occlusion of the lumen and in turn causes infarction of the area supplied by this artery, especially in cases of tentorium notch hernia where the PCA on the affected side is compressed, which is likely to trigger occipital infarction. Midline displacement in cases of brain hernia or hematoma masses can directly cause compression of the MCA, leading to infarction of the area supplied by this artery. In cases of

Table 4 Characteristics in the ROC Curves of the Six Independent Risk Factors

\begin{tabular}{|l|c|c|c|c|}
\hline Variables & Auc & Se & $95 \%$ ci & $\begin{array}{c}\text { Cut-Off } \\
\text { Value }\end{array}$ \\
\hline Admission GCS & 0.669 & 0.0265 & $0.612-0.722$ & NA \\
\hline Skull base fractures & 0.599 & 0.0458 & $0.541-0.656$ & NA \\
\hline Traumatic SAH & 0.666 & 0.0303 & $0.610-0.720$ & NA \\
\hline Brain herniation & 0.58 & 0.0453 & $0.522-0.637$ & NA \\
\hline Hypotensive shock & 0.617 & 0.0462 & $0.559-0.672$ & NA \\
\hline $\begin{array}{l}\text { Decompressive } \\
\text { craniectomy }\end{array}$ & 0.548 & 0.0461 & $0.490-0.606$ & NA \\
\hline
\end{tabular}

Abbreviations: $\mathrm{AUC}$, area under the curve; $\mathrm{SE}$, standard error; $\mathrm{Cl}$, confidence interval; NA, not available. cerebral falx hernia, compression of the cingulate gyrus leads to bending deformation of the ACA and thus infarction of the area supplied by this artery. ${ }^{5,6}$ Therefore, the occurrence of brain hernia is closely related to PTCI. In this study, we found that the incidence rates of PTCI are $18.57 \%(13 / 70)$ in patients with cerebral herniation and $8.37 \%(19 / 227)$ in those without cerebral herniation; the difference is statistically significant $(\mathrm{p}<0.05)$. However, vascular compression is only one of the causes of PTCI. Other mechanisms include vasospasm (Figure 3), embolism, a hypercoagulable state after TBI, and blunt cerebrovascular injury (BCVI). Among these mechanisms, BCVI has been considered an independent risk factor for PTCI. ${ }^{7-10}$ Previous studies showed that the probability of PTCI occurrence increases with age. ${ }^{11,12}$ Borsotti reported that patients older than 50 years have a lower risk of PTCI and argued that elderly individuals are less prone to cerebral vasospasm since cerebral arteriosclerosis has already occurred, while a certain degree of brain atrophy alleviates brain compression, displacement, and edema. ${ }^{13}$ In addition, men with TBI have been reported to be more likely to develop PTCI than women. ${ }^{12}$ However, our data show no significant difference in the PTCI incidence among patients of different ages and sexes ( $p>0.05)$.

Tian et $\mathrm{al}^{14}$ showed that a lower GCS score (ie, a more severe injury) corresponds to a higher probability of PTCI, which is consistent with our results showing incidence rates of $15.87 \%$ and $1.85 \%$ for patients with GCS scores of 3-8 and 9-12, respectively; the difference is statistically significant $(p<0.01)$. Skull base fractures have a high probability of being accompanied by vascular mechanical damage, which can likely result in the formation of intramural hematomas or dissecting aneurysms, leading to vascular stenosis and thus cerebral infarction; at the same time, vascular damage stimulates the release of tissue thromboplastin, which activates the coagulation system to form a thrombus, leading to cerebral infarction. ${ }^{15}$ We found that the incidence of PTCI in patients with skull base fractures is $18.84 \%$, which is considerably higher than that of patients without such fractures $(p<0.05)$. Rowland et al $^{16}$ conducted a prospective study on 130 patients with closed brain injuries with SAH confirmed by $\mathrm{CT}$ and found that the incidence of PTCI is 7.7\% 4-16 days after injury and that the incidence rates of PTCI in patients with mild and severe SAH are 3.0\% (3/101) and 24.1\% (7/ 29), respectively, confirming that $\mathrm{SAH}$ is a risk factor for PTCI and that the main location of SAH is closely related to the corresponding location of severe vasospasm. These findings were also confirmed in our study. 

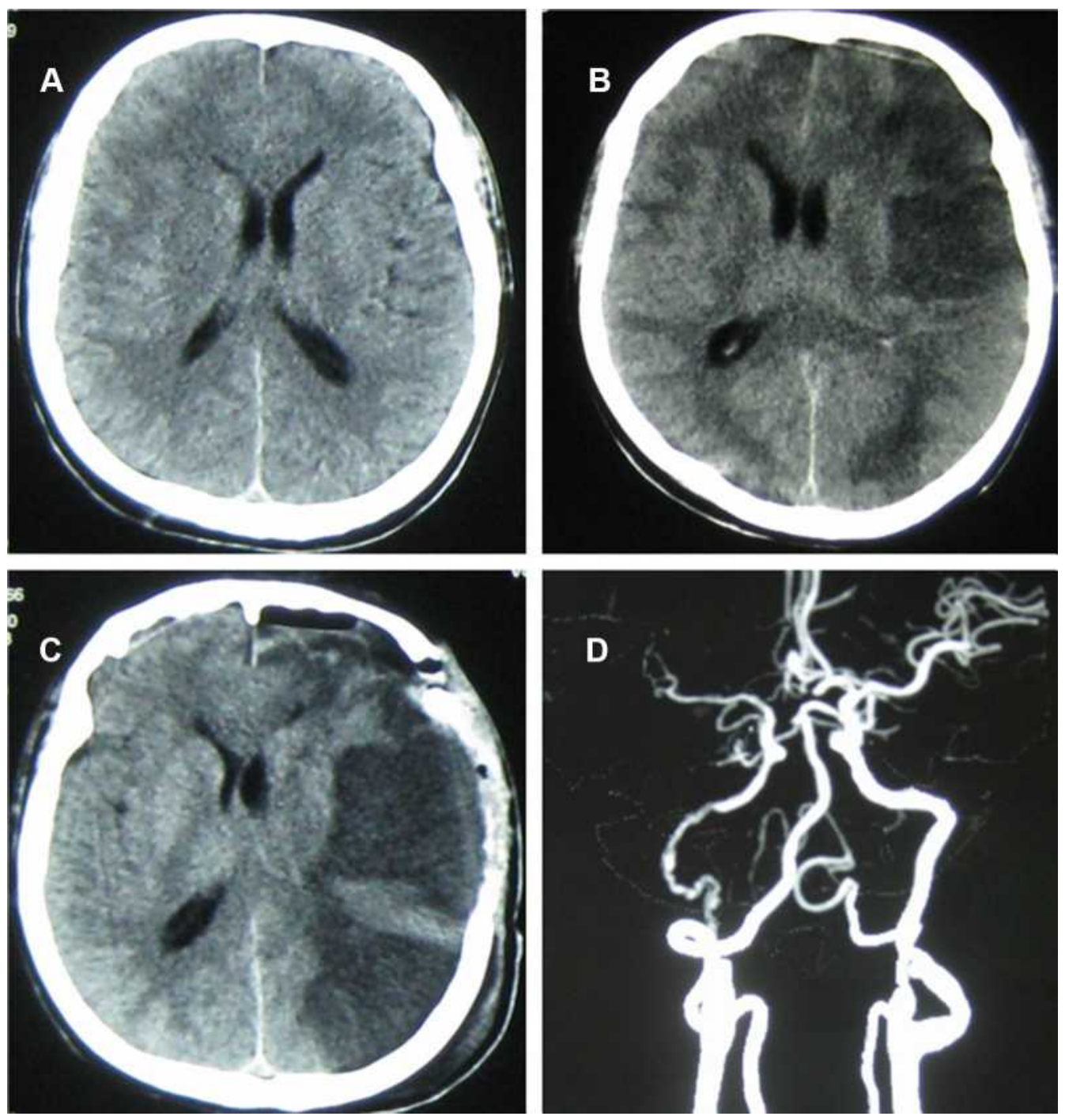

Figure 3 Posttraumatic cerebral infarction related to vasospasm. CT image of a 44-year-old man with right frontal lobe contusion and subarachnoid hemorrhage on admission after a car accident (A). The patient developed aphasia and right hemiplegia the next day with large area cerebral infarction in the left hemisphere, lateral ventricle compression and midline shift (B). Postsurgical CT scan after decompressive craniectomy revealed well-marginated low density in the area of the left middle cerebral artery (C). Vasospasm was detected by CT Angiography(D).

Most patients with craniocerebral injuries have multiple injuries. The decompensation caused by excessive blood loss or increased intracranial pressure leads to blood pressure drops and then insufficient cerebral perfusion, which can cause cerebral infarction and aggravate the craniocerebral injury. Tian et a ${ }^{14}$ noticed that a decrease in systolic blood pressure is closely related to the occurrence of PTCI. In this study, the incidence of PTCI in patients with hypotensive shock is $20.59 \%$ (14/68), which is substantially and significantly $(\mathrm{p}<0.01)$ higher than that in patients without hypotensive shock.

Some studies ${ }^{17-19}$ showed that decompressive craniectomy can reduce the effects of high intracranial pressure on brain tissues and vessels, which improves cerebral blood perfusion and the patient survival rate while lowering the incidence of PTCI. However, decompressive craniectomy itself is harmful to brain tissues. Moreover, the incisional hernia formed by bulging brain tissue after decompressive craniectomy increases the incidence of PTCI. Uhl et $\mathrm{al}^{20}$ found that for patients aged 50 years and younger, decompressive craniectomy can yield encouraging results, but for patients aged older than 50 years, the effectiveness of this surgery is controversial and requires confirmation by a prospective randomized study. In this study, the incidence of PTCI in patients who underwent decompressive craniectomy is $14.38 \%(23 / 160)$, which is significantly different from that in patients who had not undergone this procedure $(\mathrm{p}<0.05)$. 
Systemic inflammation response runs through the whole process of secondary brain injury. ${ }^{21,22}$ Some studies $^{15,23}$ found that injured nerve cells after TBI can produce brain derived microvesicle (BDMV), which have high procoagulant activity and potential pro-inflammatory activity, can lead to cerebral infarction after traumatic brain injury. Because of the retrospective nature of this study design, we were limited by the data that were collected as standard of treatment. Future prospective, multicenter studies should allow for the collection of other possible factors that may influence systemic inflammation response. C-reactive protein (CRP) and BDMV could be a viable addition to biomarker panels for risk factors of PTCI.

\section{Conclusion}

PTCI is a common clinical complication that seriously affects the prognosis of patients; therefore, early prevention, diagnosis, and treatment are very important. Low GCS score, skull base fractures, traumatic SAH, brain herniation, hypotensive shock, and craniectomy are risk factors for the occurrence of PTCI, while age and sex are not significantly correlated with the occurrence of PTCI.

\section{Abbreviations}

ACA, anterior cerebral artery; AUC,area under the curve; BCVI, blunt cerebrovascular injury; BDMV, brain derived microvesicle; $\mathrm{CI}$, confidence interval; $\mathrm{CT}$, computed tomography; CRP,C-reactive protein; GCS, admission Glasgow Coma Scale; MCA, middle cerebral artery; MRI, magnetic resonance imaging; NA,not available; OR,odds ratio; PCA, posterior cerebral artery; PTCI, Posttraumatic cerebral infarction; ROC, receiver operating characteristic; SAH, subarachnoid hemorrhage; SE,standard error; TBI, traumatic brain injury.

\section{Ethics Statement}

We obtained ethical approval to carry out this study from the Institutional Review Board (IRB) of The First Affiliated Hospital of USTC, Hefei, Anhui, China (Approval Number:2021-RE-031). All patients or their relatives provided written informed consent for this research, which was carried out in compliance with the Helsinki Declaration. All individual information was strictly kept confidential and anonymous in the manuscript.

\section{Acknowledgments}

This study was supported by grants from Anhui Natural Science Foundation (No:2008085MH250), Special Fund for basic scientific research in Central University (No: WK9110000123) and Special Fund Project for Guiding Local Science and Technology Development by the Central Government (No: 2019b07030001).

\section{Disclosure}

The authors report no conflicts of interest in this work.

\section{References}

1. Brain Trauma Foundation; American Association of Neurological Surgeons; Congress of Neurological Surgeons; Joint Section on Neurotrauma and Critical Care, AANS/ CNS, Bratton SL, Chestnut RM, Ghajar J, et al. Guidelines for the management of severe traumatic brain injury. XV. Steroids. $J$ Neurotrauma. 2007;24(Suppl 1):S91-5.

2. Mirvis SE, Wolf AL, Numaguchi Y, et al. Posttraumatic cerebral infarction diagnosed by CT: prevalence, origin, and outcome. AJR Am J Roentgenol. 1990;154(6):1293-1298.

3. Latronico N, Piva S, Fagoni N, et al. Impact of a posttraumatic cerebral infarction on outcome in patients with TBI: the Italian multicenter cohort INCEPT study. Crit Care. 2020;24(1):33.

4. Liu S, Wan X, Wang S, et al. Posttraumatic cerebral infarction in severe traumatic brain injury: characteristics, risk factors and potential mechanisms. Acta Neurochir (Wien). 2015;157(10):1697-1704.

5. Su TM, Lan CM, Lee TH, et al. Posttraumatic cerebral infarction after decompressive craniectomy for traumatic brain injury: incidence, risk factors and outcome. Turk Neurosurg. 2017;23.

6. Tawil I, Stein DM, Mirvis SE, et al. Posttraumatic cerebral infarction: incidence, outcome, and risk factors. J Trauma. 2008;64(4):849-853.

7. Al-Mufti F, Amuluru K, Lander M, et al. Low glasgow coma score in traumatic intracranial hemorrhage predicts development of cerebral vasospasm. World Neurosurg. 2018;120:e68-e71.

8. Zhang J, Zhang F, Dong JF. Coagulopathy induced by traumatic brain injury: systemic manifestation of a localized injury. Blood. 2018;131 (18):2001-2006.

9. Grigorian A, Kabutey NK, Schubl S, et al. Blunt cerebrovascular injury incidence, stroke-rate, and mortality with the expanded Denver criteria. Surgery. 2018;164(3):494-499.

10. LaRiccia AK, Wolff TW, Magee DJ, et al. Variability of radiological grading of blunt cerebrovascular injuries in trauma patients. Int J Crit Illn Inj Sci. 2020;10(2):81-87.

11. Ivanusa M, Ivanusa Z. Risk factors and in-hospital outcomes in stroke and myocardial infarction patients. BMC Public Health. 2004;5(4):26.

12. Desai M, Morris NA. Prolonged post-traumatic vasospasm resulting in delayed cerebral ischemia after mild traumatic brain injury. Neurocrit Care. 2018;29(3):512-518.

13. Borsotti F, Cossu G, Bartolini B, et al. Cerebral vasospasm following mild traumatic brain injury: a silent killer? Am J Med. 2020;133 (4):441-443.

14. Tian HL, Geng Z, Cui YH, et al. Risk factors for posttraumatic cerebral infarction in patients with moderate or severe head trauma. Neurosurg Rev. 2008;31(4):431-436.

15. Wu Y, Liu W, Zhou Y, et al. von Willebrand factor enhances microvesicle-induced vascular leakage and coagulopathy in mice with traumatic brain injury. Blood. 2018;132(10):1075-1084. 
16. Rowland MJ, Hadjipavlou G, Kelly M, et al. Delayed cerebral ischaemia after subarachnoid haemorrhage: looking beyond vasospasm. Br J Anaesth. 2012;109(3):315-329.

17. Rahmanian A, Seifzadeh B, Razmkon A, et al. Outcome of decompressive craniectomy in comparison to nonsurgical treatment in patients with malignant MCA infarction. Springerplus. 2014;28 (3): 115 .

18. Goedemans T, Verbaan D, Coert BA, et al. Outcome after decompressive craniectomy for middle cerebral artery infarction: timing of the intervention. Neurosurgery. 2020;86(3):E318-E325.

19. Reinink H, Jüttler E, Hacke W, et al. Surgical decompression for space-occupying hemispheric infarction: a systematic review and individual patient meta-analysis of randomized clinical trials. JAMA Neurol. 2020;12:e203745.
20. Uhl E, Kreth FW, Elias B, et al. Outcome and prognostic factors of hemicraniectomy for space occupying cerebral infarction. $J$ Neurol Neurosurg Psychiatry. 2004;75(2):270-274.

21. Corps KN, Roth TL, McGavern DB. Inflammation and neuroprotection in traumatic brain injury. JAMA Neurol. 2015;72(3):355-362.

22. Shetty T, Cogsil T, Dalal A, et al. High-Sensitivity C-reactive protein: retrospective study of potential blood biomarker of inflammation in acute mild traumatic brain injury. J Head Trauma Rehabil. 2019;34 (3):E28-E36.

23. Tian Y, Salsbery B, Wang M, et al. Brain-derived microparticles induce systemic coagulation in a murine model of traumatic brain injury. Blood. 2015;125(13):2151-2159.

\section{Publish your work in this journal}

Therapeutics and Clinical Risk Management is an international, peerreviewed journal of clinical therapeutics and risk management, focusing on concise rapid reporting of clinical studies in all therapeutic areas, outcomes, safety, and programs for the effective, safe, and sustained use of medicines. This journal is indexed on PubMed Central, CAS,
EMBase, Scopus and the Elsevier Bibliographic databases. The manuscript management system is completely online and includes a very quick and fair peer-review system, which is all easy to use. Visit http://www.dovepress.com/testimonials.php to read real quotes from published authors. 